\title{
Héritabilité de la résistance à la fusariose de l'épi et sélection récurrente dans une population de blé tendre
}

\author{
L Saur, M Trottet \\ avec la collaboration technique de JY Morlais \\ INRA, station d'amélioration des plantes, Domaine de la Motte-au-Vicomte, BP 29, 35650 Le Rheu, France \\ (Reçu le $1^{\text {er }}$ janvier 1991; accepté le 26 février 1992)
}

\begin{abstract}
Résumé - Cinquante-six familles $S_{1}$ et leurs descendances $S_{2}$ issues d'une population de blé tendre en sélection récurrente utilisant un gène de stérilité mâle dominant ont été évaluées pour leur résistance à la fusariose de l'épi. Les tests de résistance ont été réalisés au champ avec contamination artificielle et ont été répétés sur 2 campagnes culturales pour les $S_{1}$. Les symptômes sur épi et sur grain ont été notés, ainsi que l'effet de la maladie sur le rendement et le poids de 1000 grains par comparaison avec un témoin non inoculé. Une corrélation significative a été trouvée entre les symptômes et la diminution du poids de 1000 grains $(r=0,75)$. L'interaction génotype $\times$ année est significative mais le coefficient de corrélation entre les 2 années est élevé ( $r=0,79$ pour les symptômes sur grain). Les valeurs de l'héritabilité au sens large pour les différents caractères sont comprises entre 0,8 et 0,9 et justifient la sélection précoce pour la résistance à la fusariose de l'épi menée sur cette population. La faiblesse de l'héritabilité au sens strict entre $S_{1}$ et $S_{2}$ suggère d'utiliser les individus frères des individus testés et non leurs descendants dans le schéma de sélection récurrente.
\end{abstract}

résistance / Fusarium culmorum/blé / sélection récurrente / sélection précoce / héritabilité

Summary - Heritability and recurrent selection for resistance to Fusarium head blight in winter wheat. Fifty-six $S_{1}$ families and their $S_{2}$ progeny from a winter wheat population with genic male sterility were evaluated for reaction to Fusarium head blight. Families were tested in the field using artificial inoculation. Tests were repeated over 2 years for $S_{1}$ families. Disease severity was scored on spikes and seeds. The effect of the disease on yield and thousand grain weight was measured using uninoculated control plots. A significant correlation was found between disease severity and thousand grain weight decrease (table I). Despite significant year $\times$ genotype interaction (table II), correlation coefficients between the 2 years for disease evaluation on $S_{1}$ families were high ( $r=0.79$ for severity in seeds). Broad sense heritabilities were from 0.8 to 0.9 (table III) and justify early selection for Fusarium resistance in the population. Because of low $S_{1}-S_{2}$ heritability (table V), using sibs of tested plants instead of offspring is suggested for the recurrent selection scheme.

resistance / Fusarium culmorum / wheat / recurrent selection / heritability

Nouvelle adresse: INRA, SRIV, Domaine de la Motte-au-Vicomte, BP 29, 35650 Le Rheu, France 


\section{INTRODUCTION}

La fusariose de l'épi causée par Fusarium culmorum (WG Smith), est une maladie du blé pour laquelle on ne connaît pas de haut niveau de résistance (Mielke, 1988; Snijders, 1990; Saur, 1991). La sélection généalogique ou en bulk, à partir de croisements simples entre 2 ou 4 parents, suivis d'autofécondation, n'a pas permis d'obtenir de lignées supérieures aux meilleures variétés connues. La sélection récurrente, en augmentant le nombre de recombinaisons par unité de temps, permet d'exploiter une base génétique beaucoup plus large et augmente les chances d'obtenir des génotypes nouveaux (Allard, 1960; Gallais, 1981). Les exemples de sélection récurrente dans des populations de plantes autogames sont nombreux (Kervella et al, 1991). Thomas et al (1991) ont présenté une population de blé tendre issue d'un croisement pyramidal à 16 parents destinée à des études méthodologiques concernant la sélection récurrente. Des gènes de stérilité mâle existent chez le blé et permettent de faciliter les recroisements dans les populations (Deng et Gao 1982; Thompson, 1983). Trottet (1988) a décrit une population créé a avec un gène de stérilité mâle récessif et un schéma de sélection récurrente pour améliorer la résistance à la septoriose causée par Leptosphaeria nodorum. D'autres populations, utilisant un gène de stérilité mâle dominant et faisant intervenir un grand nombre de géniteurs, ont été créées à la station d'amélioration des plantes INRA de Rennes dans le but d'obtenir des génotypes combinant des résistances à plusieurs maladies, dont la fusariose de l'épi.

L'application à ces populations de schémas de sélection récurrente à cycle court nécessite une évaluation précoce des génotypes hétérozygotes avant le retour à l'homozygotie par autofécondation. Aucun test d'évaluation de la résistance à la fusariose de l'épi n'est disponible pour les plantes prises individuellement. Le jugement est réalisé au champ après contamination artificielle, sur les familles issues de plantes ayant subi une seule année d'autofécondation (familles $S_{1}$ ). Sachant que le développement de la maladie dépend du stade de la plante et des conditions climatiques au moment de la contamination (Saur et Trottet, 1981), il est utile de savoir dans quelle mesure les disjonctions de précocité à l'intérieur des familles diminuent la précision de l'estimation de la valeur génétique par rapport à ce que l'on peut obtenir avec des lignées homozygotes.

Selon le schéma de sélection récurrente utilisé, les unités croisées sont les frères ou les descendants des unités sélectionnées. Pour choisir un schéma de sélection adapté au caractère à sélectionner, il est nécessaire de connaître la relation entre la valeur propre des familles testées et la valeur des descendants en autofécondation.

Le but de cette étude est d'évaluer l'influence de l'interaction génotype-milieu sur l'estimation de la valeur génétique de familles issues d'une population et d'étudier la relation entre le comportement de familles $S_{1}$ et de leur descendance $S_{2}$.

\section{MATÉRIEL ET MÉTHODES}

Une population de blé tendre possédant un gène dominant de stérilité mâle a été créée à partir de 190 lignées parentales. Les géniteurs ont été introduits par croisements dirigés sur les plantes mâle stériles de la variété Chris possédant un gène de stérilité mâle dominant (Sasakuma et al, 1978). Le cycle de sélection récurrente est de 3 ans. La population en disjonction pour la stérilité mâle est semée à faible densité ( $40 \mathrm{grains} / \mathrm{m}^{2}$ ) afin de pouvoir individualiser les plantes. Les grains récoltés sur les plantes stériles servent à reproduire la population et les plantes fertiles $S_{0}$ sont récoltées individuellement. Pour chaque plante fertile récoltée, une partie des grains est utilisée pour réaliser des essais destinés à évaluer la résistance à quelques maladies dont la fusariose de l'épi, le reste étant mis en réserve pour une utilisation ultérieure. La sélection est basée sur un index combinant les résultats des différents essais. Cet index est obtenu par combinaison linéaire des valeurs phénotypiques pour les caractères sélectionnés, les valeurs des coefficients étant attribuées a priori pour chaque caractère. Plusieurs index sont calculés successivement en faisant varier les pondérations et nous conservons celui pour lequel le progrès génétique sur chaque caractère correspond le mieux à l'objectif fixé. Le poids le plus élevé est donné à la résistance aux maladies de fin de végétation (fusariose de l'épi, septoriose, rouille brune). Pour les familles sélectionnées, les grains en réserve sont semés l'année suivante et servent de pollinisateurs dans la population.

Les grains récoltés sur 56 plantes fertiles $S_{0}$ en 1988 ont été divisés en 2 lots pour réaliser des essais sur familles $S_{1}$, sur 2 années culturales 1989 et 1990 . En 1989 , pour chaque famille $S_{1}$, 
les grains issus des parcelles non contaminées de l'essai ont été récoltés pour former les familles $S_{2}$. Ces familles ont été mises en essai en 1990.

Les essais de résistance à la fusariose de l'épi ont été réalisés en plein champ. Chaque famille a été contaminée par pulvérisation d'une suspension de spores d'un isolat de Fusarium culmorum à la floraison des premiers épis. Une deuxième contamination a été réalisée 2 jours après pour tenir compte de la variation de précocité intrafamille.

Un dispositif à 2 répétitions avec un traitement contaminé et un traitement non contaminé a été utilisé. La parcelle élémentaire était un poquet de 15 à 20 plantes avec un maillage de $50 \times 50 \mathrm{~cm}$ entre poquets.

Pour chaque famille, le pourcentage d'épillets attaqués par $F$ culmorum a été estimé visuellement 350 et $450^{\circ} \mathrm{C} \cdot \mathrm{j}$ après la contamination (N350, N450). À la récolte, les grains couverts de mycélium blanc rose (grains fusariés: Gf) ont été dénombrés sur un échantillon de 500 grains.

L'effet du parasite sur le rendement a été mesuré par le rapport du poids de grain de la parcelle contaminée au poids de grain de la parcelle non contaminée exprimé en pourcentage: (Pdsc\%). L'effet sur le poids de 1000 grains est calculé de la même façon ( $P m g c \%$ ).

Les analyses de variance ont été réalisées pour les familles $S_{1}$ sur les 2 années. Le modèle utilisé est un modèle à 3 facteurs (année, famille et répétition), le facteur répétition étant hiérarchisé sous le facteur année. Les héritabilités au sens large ont été calculées à partir de la variance interfamille $V f$ et de la variance résiduelle $V r$ : $h^{2}=V f /(V f+V r / 2)$. Les intervalles de confiance ont été évalués selon la méthode proposée par Tai (1989). Les héritabilités au sens strict ont été estimées à partir de la régression parents-descendants pondérée par la consanguinité des parents, soit pour des $S_{1}: h^{2}=2 / 3 b$ (Smith et Kinman, 1965).

La formule utilisée pour le calcul du progrès génétique attendu a été la suivante: $\delta G=i h \sigma_{g}$ (Gallais, 1984) où $i$ est l'intensité de sélection (différence entre la moyenne des individus sélectionnés et la moyenne générale, rapportée à l'écart type), $h^{2}$ est l'héritabilité et $\sigma_{g}^{2}$ la variance génétique ou variance interfamille.

La réponse attendue à la sélection pour un caractère 2 lorsque la sélection est pratiquée sur un caractère 1 a été calculée par la formule $\delta G_{2}=i h_{1}$ $\sigma_{2} r$, où $h_{1}$ est l'héritabilité du caractère $1, \sigma_{2}^{2}$ la variance génétique du caractère 2 et $r$ la corrélation génétique entre les 2 caractères.

Étant donné que dans le schéma de sélection utilisé, seules les plantes mâles sont sélectionnées pour la résistance à la fusariose, le progrès génétique a été pondéré par un coefficient de contrôle de la parenté de valeur $\theta=0,5$.
Des essais de comparaison de lignées comprenant 40 variétés et lignées en fin de sélection étaient implantés chaque année dans la même parcelle selon le même dispositif. Les résultats de ces essais ont été utilisés pour comparer la précision des estimations sur des familles en disjonction et sur des lignées pures.

\section{RÉSULTATS ET DISCUSSION}

\section{Relation entre les variables étudiées}

Les coefficients de corrélation entre les différents caractères mesurés sur les valeurs moyennes des familles $S_{1}$ sur 2 ans sont présentés dans le tableau I. Ils sont tous significatifs au seuil 0,01 .

Le coefficient de corrélation entre les notes sur épi 350 et $450^{\circ} \mathrm{Cj}$ après la contamination est de 0,96. La liaison entre chacune des 2 notations et leur moyenne $\left(N_{\text {moy }}\right)$ étant très forte $(r=0,99)$, nous utiliserons la note moyenne dans la suite de la présentation des résultats. La valeur élevée du coefficient de corrélation entre les symptômes sur grains et en végétation montre que l'estimation visuelle de la quantité de symptômes, sur un ensemble d'épis de stades différents en raison de la disjonction de précocité intrafamille, est possible.

Tableau I. Coefficients de corrélation entre les caractères évaluant la résistance à la fusariose de l'épi calculés à partir des valeurs moyennes sur 2 ans des familles $S_{1}$.

\begin{tabular}{|c|c|c|c|c|c|c|}
\hline & Pdsc\% & Pmgc\% & Gf & $\mathrm{N}_{\text {moy }}$ & N350 & N450 \\
\hline$P d s c \%$ & 1,00 & & & & & \\
\hline$c \%$ & 0,58 & 1,00 & & & & \\
\hline$G t$ & $-0,45$ & $-0,78$ & 1,00 & & & \\
\hline$N_{\text {moy }}$ & $-0,57$ & $-0,76$ & 0,77 & 1,00 & & \\
\hline & $-0,56$ & & 0,75 & 0,99 & 1,00 & \\
\hline V450 & $-0,56$ & $-0,75$ & 0,76 & 0,99 & 0,96 & 1,000 \\
\hline
\end{tabular}

Pdsc\%: Poids de grain de la parcelle contaminée/poids de grain du témoin non contaminé (\%), Pmgc\%: Poids de 1000 grains de la parcelle contaminée / poids de 1000 grains du témoin non contaminé (\%); Gf: Nombre de grains fusariés (\%); $N_{\text {moy }}$ : Note moyenne de maladie sur épi; $N 350$ : Nole de maladie sur épi $350^{\circ} \mathrm{C}$ j après la contamination; N450: Note de maladie sur épi $450^{\circ} \mathrm{C} j$ après la contamination. 
Les corrélations entre les symptômes en végétation ou sur grains et les mesures de l'effet du parasite sur le rendement sont significatives. Les valeurs des coefficients sont plus faibles pour le $P d s c \%$ que pour le $P m g c \%$. Ces résultats peuvent être expliqués par la faible précision de l'estimation du rendement en grain sur des parcelles très petites. Ceci explique de même la faiblesse du coefficient de corrélation entre le $P m g c \%$ et le $P d s c \%$. En effet, dans les dispositifs permettant d'évaluer le rendement avec plus de précision, le coefficient de corrélation entre ces 2 variables est élevé (Saur, 1991). L'effet de la maladie sur le rendement peut alors être bien estimé par l'effet de la maladie sur le poids de 1000 grains.

La mesure de l'effet de la maladie sur le poids de 1000 grains évalue la combinaison de 2 aspects du comportement du génotype: sa résistance et, s'il est attaqué, l'influence de cette attaque sur la formation du grain ou tolérance. La valeur du coefficient de corrélation entre les symptômes et la diminution du poids de 1000 grains est élevée dans cette population. Les valeurs du Pmgc\% sont donc peu différentes pour des génotypes présentant la même quantité de symptômes. Ceci indique que la variabilité pour la tolérance est faible.

\section{Effet de l'année}

Les résultats de l'analyse de variance sur les $S_{1}$ pour les 2 années sont présentés dans le tableau II.

Une différence entre les 2 années, significative au seuil 0,001 , est mise en évidence pour tous les caractères sauf le Pmgc \%. Le développement de la maladie observé sur des variétés témoins a été plus important en 1990 qu'en 1989. La gravité de l'attaque s'est traduite par une diminution du nombre de grains en 1990 alors que l'effet sur le poids de 1000 grains est le même pour les 2 années.

La meilleure discrimination entre familles est donnée par la notation sur grain ( $G f$ ) pour laquelle la valeur du $F$ pour l'effet famille est la plus élevée.

L'interaction année $\times$ famille est significative au seuil 0,05 pour les notations sur grain (Gf) comme en végétation ( $N_{\text {moy }}$ ) alors qu'elle n'est pas mise en évidence pour les mesures de l'effet sur le rendement $(P d s c \%$ et $P m g c \%)$. Les variances familles sont cependant significativement supérieures aux variances de l'interaction.

Les coefficients de corrélation entre les résultats obtenus sur les familles $S_{1}$ pour les 2 années sont significatifs au seuil 0,05 pour tous les paramètres étudiés. Ils sont de 0,79 pour le nombre de grains fusariés et 0,65 pour les symptômes en végétation. Ils sont plus faibles pour les mesures sur le rendement malgré l'absence d'interaction significative famille $x$ année $(0,49$ pour le $P m g c \%$ et 0,38 pour le $P d s c \%$ ). Ceci peut s'expliquer par la moins bonne précision de l'estimation de ces caractères et donc une variance résiduelle plus élevée.

L'interaction famille $x$ année observée est donc faible malgré d'importantes différences de précocité entre les familles et un effet de l'année sur les différences de développement des génotypes: la période de contamination de la famille la plus précoce à la plus tardive s'étendait sur $10 \mathrm{j}$ en 1989 et 22 en 1990. Dans une étude sur 25 variétés sur 3 ans, Mesterhazy (1986) trouve des coefficients de corrélation entre années de 0,42 à 0,80 pour les symptômes et de 0,36 à 0,72 pour la diminution de rendement. Les disjonctions intrafamilles dans notre étude ne se traduisent donc pas par une diminution importante de la corrélation entre années par rapport à des lignées pures.

Tableau II. Analyse de variance sur les résultats des essais fusariose de l'épi pour 56 familles $S_{1}$ sur 2 années d'expérimentation.

\begin{tabular}{lrrrrrrrrr}
\hline & \multirow{2}{*}{ ddl } & \multicolumn{2}{c}{$\mathrm{Pdsc} \%$} & \multicolumn{2}{c}{$\mathrm{Pmgc} \%$} & \multicolumn{2}{c}{ Gf } & \multicolumn{2}{c}{$\mathrm{N}_{\text {may }}$} \\
& & \multicolumn{1}{c}{$\mathrm{F}^{a}$} & \multicolumn{1}{c}{$\mathrm{P}^{b}$} & \multicolumn{1}{c}{$\mathrm{F}$} & \multicolumn{1}{c}{$\mathrm{P}$} & \multicolumn{1}{c}{$\mathrm{F}$} & $\mathrm{P}$ & \multicolumn{1}{c}{$\mathrm{F}$} & \multicolumn{1}{c}{$\mathrm{P}$} \\
\hline Année & $1 / 55$ & 39,9 & $<0,001$ & 0,1 & 0,717 & 78,4 & $<0,001$ & 41,2 & $<0,001$ \\
Famille & $55 / 55$ & 2,1 & 0,035 & 2,9 & $<0,001$ & 6,7 & $<0,001$ & 4,6 & $<0,001$ \\
Interaction & $55 / 110$ & 1,1 & 0,301 & 1,2 & 0,188 & 2,3 & $<0,001$ & 1,6 & 0,022 \\
famille $\times$ année & & & & & & & & &
\end{tabular}

\footnotetext{
${ }^{a}$ : valeur du $F$ de Fisher; ${ }^{b}$ : probabilité d'observer une valeur du $F$ supérieure sous hypothèse nulle.
} 


\section{Estimation de l'héritabilité au sens large}

Les valeurs des héritabilités sont élevées pour tous les caractères retenus (tableau III). La résistance mesurée sur grain $(G f)$ est le caractère qui présente la plus forte héritabilité.

Ces valeurs obtenues sur des familles en disjonction ne sont pas très inférieures à celles que nous avons obtenues avec les lignées homozygotes. Sur l'échantillon de 40 lignées comprenant des témoins très sensibles et assez résistants, nous avons trouvé avec 2 répétitions des valeurs de l'héritabilité de 0,86 en 1989 et 0,85 en 1990 pour le Pmgc\%. Snijders (1990), sur 32 variétés lignées pures sur une année et 54 sur une autre, trouve des valeurs de 0,66 et 0,96 pour la diminution du poids de 1000 grains et 0,82 et 0,86 pour les symptômes.

Le tableau IV présente le progrès génétique attendu pour un taux de sélection de $20 \%$. La note moyenne de sensibilité en végétation $\left(N_{\text {moy }}\right)$ serait diminuée de 0,5 et le $P m g c \%$ augmenterait de 7 à $8 \%$. Dans l'hypothèse d'une sélection sur la note de sensibilité, le progrès indirect sur les autres paramètres de mesure de la résistance resterait satisfaisant.

Tableau III. Valeur de l'héritabilité au sens large sur les familles $S_{1}$ pour les caractères évaluant la résistance à la fusariose de l'épi.

\begin{tabular}{cccccc}
\hline & & Pdsc\% & Pmgc\% & Gf & $N_{\text {moy }}$ \\
\hline 1989 & $h^{2}$ & 0,77 & 0,84 & 0,93 & 0,92 \\
& $l c$ & $0,60-0,86$ & $0,73-0,91$ & $0,89-0,96$ & $0,86-0,95$ \\
1990 & $h^{2}$ & 0,79 & 0,82 & 0,96 & 0,87 \\
& $l c$ & $0,64-0,88$ & $0,69-0,89$ & $0,92-0,97$ & $0,78-0,92$ \\
\hline
\end{tabular}

$h^{2}$ : valeur de l'héritabilité; $l c$ : intervalle de confiance au seuil 0,05 .

Tableau IV. Progrès génétique attendu dans la population avec un taux de sélection de $20 \%$ pour les caractères mesurant la résistance à la fusariose de l'épi.

\begin{tabular}{llrrrr}
\hline & & Pdsc\% & Pmgc\% & Gf & $N_{\text {moy }}$ \\
\hline 1989 & $\delta G_{d}$ & 13,8 & 6,1 & 43 & 0,62 \\
& $\delta G_{i}$ & 5,5 & 4,7 & 37 & \\
1990 & $\delta G_{d}$ & 11,0 & 6,5 & 38 & 0,50 \\
& $\delta G_{i}$ & 6,6 & 4,3 & 29 & \\
\hline
\end{tabular}

$\delta G_{d}$ : Progrès génétique direct attendu pour une sélection sur le caractère; $\delta G_{i}$ : Effet indirect d'une sélection sur la notation en végétation $N_{\text {moy }}$.

\section{Estimation de l'héritabilité au sens strict}

Les héritabilités au sens strict évaluées avec les résultats obtenus sur les familles $S_{1}$ et leurs descendances $S_{2}$, dans les essais réalisés la même année, sont données dans le tableau $\mathrm{V}$.

Les valeurs des héritabilités sont faibles pour une sélection familiale, sauf pour la mesure du nombre de grains fusariés pour laquelle elle est de 0,58 . Les 80 grains destinés au test $S_{2}$ ont été échantillonnés dans le produit en mélange de 20 plantes $S_{1}(1500$ grains environ). Cet échantillon ne représente qu'une partie des individus testés en $S_{1}$.

\section{CONCLUSION}

Pour la gamme de précocité couverte par notre population, et malgré la disjonction pour la précocité intrafamille, l'interaction famille $\times$ année est faible. Ceci nous permet de continuer à envisager l'évaluation de la valeur des familles dans des tests au champ, à condition de contaminer et noter chaque famille au même stade. L'implantation de variétés témoins de précocité différente et de comportement connu est cependant nécessaire pour vérifier que les conditions ont été favorables au développement de la maladie sur tous les groupes de précocité.

Les valeurs élevées de l'héritabilité au sens large montrent que le dispositif restreint que nous avons utilisé de 2 répétitions de 15-20 plantes est suffisant pour l'estimation de la valeur génétique des familles $S_{1}$. La résistance à la fusariose de l'épi peut être mesurée par le pourcentage d'épillets fusariés estimé visuellement pour chaque famille au même stade de développement. Cette notation demande moins de travail que la notation sur grain et permet une acquisition précoce des résultats. La faible variabilité pour la tolérance dans cette population ne justifie pas la me-

Tableau V. Valeur de l'héritabilité au sens strict entre les $S_{2}$ et les $S_{1}$ pour les caractères évaluant la résistance à la fusariose de l'épi.

\begin{tabular}{lcccc}
\hline & Pdsc\% & Pmgc\% & Gf & $N_{\text {moy }}$ \\
\hline$h^{2} s s$ & 0,23 & 0,33 & 0,58 & 0,35 \\
$l c$ & $0,14-0,32$ & $0,23-0,43$ & $0,52-0,64$ & $0,29-0,42$
\end{tabular}

$h^{2} s s$ : valeur de l'héritabilité au sens strict; $l c$ : intervalle de confiance au seuil 0,05 . 
sure de l'effet de la maladie sur le poids de 1000 grains. Les mesures sur les grains comprenant la récolte, les pesées et les comptages sont coûteuses et les résultats doivent être disponibles rapidement pour la préparation des semis de la campagne suivante. Dans un schéma de sélection récurrente, il est préférable de tester un plus grand nombre de familles $S_{1}$ et il est plus facile d'augmenter le nombre de notations en végétation que le nombre de parcelles récoltées.

Dans certains schémas de sélection récurrente avec tests sur famille $S_{1}$, quelques plantes par famille sont sélectionnées en pépinière sur les caractères visibles sur plantes individuelles. Pour les familles $S_{1}$ retenues sur les résultats des tests, les grains $S_{2}$ issus des plantes sélectionnées en pépinière sont semés comme pollinisateurs l'année suivante. Pour la résistance à la fusariose de l'épi, les valeurs des héritabilités au sens strict entre $S_{1}$ et $S_{2}$ montrent que l'utilisation pour les recroisements dans la population de plantes $S_{1}$ sœurs de celles mises en essai est préférable à l'utilisation d'un petit échantillon de descendants $S_{2}$ récoltés sur les plantes testées.

La population étudiée est soumise à une sélection multicaractère. Certains caractères sont mesurables sur plantes individuelles - résistance aux rouilles et à l'oïdium, hauteur - d'autres ne peuvent être évalués que sur famille $S_{1}$ - résistance à la fusariose, à la septoriose, rendement. La sélection des familles destinées à être utilisées comme pollinisateurs est réalisée sur les valeurs familiales pour les différents caractères. Le fait d'utiliser pour les recroisements les semences mises en réserve représentant des individus frères de ceux testés nous prive de la possibilité d'une sélection intrafamille l'année du test. II est cependant possible de pratiquer une sélection massale dans les pollinisateurs l'année du recroisement pour les caractères visibles avant la floraison comme la rouille jaune et l'oïdium et dans une certaine mesure la hauteur.

\section{RÉFÉRENCES}

Allard RW (1960) Principles of plant breeding. John Wiley Inc, New York, 485

Deng J, Gao Z (1982) Discovery and determination of a dominant male sterile gene and its importance in genetics and wheat breeding. Sci Sin (Ser B) 25 508-520
Gallais A (1981) Amélioration d'une population en vue de la création des variétés. Sel Fr 29, 5-23

Gallais A (1984) Use of indirect selection in plant breeding. In: Efficiency in plant breeding (Lange W, Zeven AC et Hogenboom G eds) Proc 10th Congr Eucarpia, Pudoc, Wageningen, 45-60

Kervella J, Goldringer I, Brabant P (1991) Sélection récurrente chez les autogames pour l'amélioration des variétés lignées pures: une revue bibliographique. Agronomie 11, 335-352

Mesterhazy A (1986) Resistance to ear fusariosis in winter wheat. Nôvenytermelés 35, 407-417

Mielke $H$ (1988) Untersuchungen über Fusarium culmorum (WGSm) Sacc als Fuss-und Ährenkrankheitserreger beim Weizen. Mitt Biol Bundesanst Land Forstwirtsch Berl Dahlem 238, $101 \mathrm{p}$

Sasakuma T, Maan SS, Williams ND (1978) EMSinduced male-sterile mutants in euplasmic and alloplasmic common wheat. Crop Sci 17 667678

Saur L (1991) Recherche des géniteurs de résistance à la fusariose de l'épi causée par Fusarium culmorum chez le blé et les espèces voisines. Agronomie 11, 535-541

Saur L, Trottet M (1981) Problèmes posés par le jugement du comportement de lignées de blé tendre et espèces voisines vis-à-vis de deux parasites de l'épi Septoria nodorum Berk et Fusarium roseum (Link) $\mathrm{Sn}$ et $\mathrm{H}$. Mise en évidence de quelques sources de résistance. $C R$ Séances Acad Agric Fr 67, 139-145

Smith JD, Kinman ML (1965) The use of parent offspring regression as an estimation of heritability. Crop Sci 5, 595-596

Snijders CHA (1990) Genetic variation for resistance to Fusarium head blight in bread wheat. Euphytica 50, 171-179

Tai GCC (1989) A new procedure to construct confidence intervals for genotypic variance and expected reponse to selection. Genome 32, 307-308

Thomas G, Rousset M, Pichon M, Trottet M, Doussinault G, Picard E (1991) Méthodologie de l'amélioration du blé tendre (Triticum aestivum L). Création par croisement et analyse d'une population artificielle à 16 parents, base de cette étude méthodologique. Agronomie 11, 359-368

Thompson RK (1983) Registration of AZ-MSFRS82RR rust resistant common wheat resistant germplasm. Crop Sci 23, 605

Trottet M (1988) Use of genic male sterility for breeding wheat lines resistant to Leptosphaeria nodorum Müller: results of a first cycle and prospects. In: Proc 7th int Wheat Genet Symp 1199-1202 